\title{
Intermediate Risk
}

National Cancer Institute

\section{Source}

National Cancer Institute. Intermediate Risk. NCI Thesaurus. Code C102402.

The potential future harm that may arise from some present action or attribute or condition is moderate. 